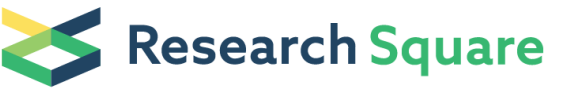

\section{Attention-Based Deep Multiple Instances Learning For Classifying Circular RNA and Other Long Non- Coding RNA}

\section{Yunhe Liu}

Institute of Biomedical Sciences, Fudan University, China

\section{Qiqing Fu}

Institute of Biomedical Sciences, Fudan University, China

\section{Xueqing peng}

Institute of Biomedical Sciences, Fudan University, China

Chaoyu Zhu

Institute of Biomedical Sciences, Fudan University, China

\section{Gang Liu}

Institute of Biomedical Sciences, Fudan University, China

Lei Liu ( $\nabla$ liulei_sibs@163.com )

Institute of Biomedical Sciences, Fudan University, China

\section{Research Article}

Keywords: circRNAs, IncRNA, network architecture

Posted Date: September 3rd, 2021

DOI: https://doi.org/10.21203/rs.3.rs-751366/v1

License: (1) This work is licensed under a Creative Commons Attribution 4.0 International License. Read Full License

Version of Record: A version of this preprint was published at Genes on December 19th, 2021. See the published version at https://doi.org/10.3390/genes12122018. 


\section{Abstract}

Circular RNA (circRNA) is a distinguishable circular formed long non-coding RNA (IncRNA), which has specific roles in transcriptional regulation, multiple biological processes. The identification of circRNA from other IncRNA is necessary for relevant research. In this study, we designed attention-based multiinstance learning (MIL) network architecture, which can be fed with raw sequence, to learn the sparse features in sequences and accomplish the identification task for circRNAs. The model outperformed previously reported models. Following the effectiveness validation of the attention score by the handwritten digit dataset, the key sequence loci underlying circRNAs recognition were obtained based on the corresponding attention score. Moreover, the motif enrichment analysis of the extracted key sequences identified some of the key motifs for circRNA formation. In conclusion, we designed a deep learning network architecture suitable for gene sequence learning with sparse features and implemented to the circRNA identification, and the network has a strong representation capability with its indication of some key loci.

\section{Introduction}

Non-coding RNAs (ncRNAs), referring to RNAs without protein-coding potential, account for the majority of RNAs. It's generally recognized that IncRNA (long non-coding RNA) is a kind of ncRNAs that is longer than 200 nucleotides, which is distinguished from other smaller ncRNA species such as miRNAs and siRNAs. IncRNA has complex biological functions such as transcriptional regulation and posttranscriptional control ${ }^{[1-3]}$. Circular RNA (circRNA) is a closed IncRNA formed by covalently closed loops. Based on current researches, circRNAs are more stable than mRNAs and play a major role as a microRNA activity modulator. CircRNAs are also correlated with the development of multiple diseases ${ }^{[3-5]}$, and can be used for disease biomarkers ${ }^{[6,7]}$. Therefore, it is vital to detect circular RNAs.

Currently, some computational approaches to distinguish circRNA from IncRNA ${ }^{[8-10]}$ have been developed with different frameworks. For example, CirRNAPL ${ }^{[11]}$ adopted the extreme learning machine based on the particle swarm optimization algorithm. CircLGB utilized a LightGBM classifier ${ }^{[12]}$. Based on an End-to-End deep learning framework, circDeep ${ }^{[13]}$ fused an RCM, ACNN-BLSTM sequence, and a conservation descriptor into high-level abstraction descriptors, and achieved an improvement with higher accuracy compared with exiting tools.

For these models mentioned above, the input of the model was not the raw sequence, but often the relevant features extracted from the predicted secondary structure ${ }^{[9,13]}$. For circDeep, a deep learning framework utilized complementary structure ${ }^{[14]}$ and conservation features. Its sequence input part did not use the full sequence and underwent a triplet transformation ${ }^{[15]}$, either. It is important to find a deep learning framework that is suitable for sequence input as well as sequence learning, to facilitate the utilization of algorithms and take advantage of the information in sequence. 
The characteristics of RNA sequences have quite different from the other sequence data like word language. We organized the differences into 3 main points. First, the RNA sequences are a combination of multiple meaningful and meaningless sequences, where the meaningful units are embedded into the entire background sequences, not like the words that form a certain grammatical structure in order ${ }^{[16]}$. An RNA typically has a large variety of functions enabled by meaningful units, such as the ability to form high-level structures and to recruit other components ${ }^{[17]}$. While learning models tend to have singular learning objectives, such as distinguishing circular RNA, which results in the meaningful units for learning model is sparse in the long sequence. Second, the length of different RNA varies greatly ${ }^{[18]}$, spans from 100 to $1,000,000 \mathrm{nt}$, suggesting that the density of the meaningful units also varies considerably. Third, the character component of the RNA sequence is relatively simple, which only contains four characters (ATGC) and the single character is meaningless. On the other hand, the composition and length of meaningful components are unknown, so the input data for learning is character instead of a meaningful word.

To address the problem mentioned above, we designed an attention-based deep encoder MIL (multiple instance learning) model (Circ-ATTEN-MIL). The MIL structure is suitable for learning sparse features ${ }^{[19}$, ${ }^{20}$, and the attention-based pooling layer can discover similarities between instances and has a stronger representation capability ${ }^{[21]}$. We applied this deep network structure to learn the identification task and achieved better accuracy and extracted high attention sequences to enrich motif, which shed light on studies regarding RNA circligase.

\section{Method}

\section{Data source:}

We extracted circRNAs sequences from the circRNADb database ${ }^{[22]}$ and other IncRNAs sequences from the GENCODE database ${ }^{[23]}$ (lincRNA, antisense, processed transcript, sense intronic, and sense overlapping) respectively. After removed sequences shorter than 200 nucleotides, we got 31939 circRNAs and 19722 other IncRNAs. The circRNA sequences were regarded as positive samples. We randomly divided the dataset into a training set (75\%), validation set (10\%), and test set (15\%).

Instances extraction by sliding window:

An RNA sequence was regarded as a bag, and instances were extracted from the sequence. For each fulllength sequence, we connected the head ( $5^{\prime}$ end) and tail ( $3^{\prime}$ end) of the sequence, set the slider window size and the sliding step, and made the slider move from the head. For each step, the sequence contained in the window was extracted as an instance, until the slider moved out of the tail of the sequence (Illustrated in Fig.1). For a sequence of a certain length, the number of instances can be calculated by the following formula. 
Number (Instances) $=\left[\frac{\text { Length (Sequence) }}{\text { Step (Slide) }}\right]_{\text {round }}$

The network structure was represented in Figure 2. We employed the encoder structure of the seq2seq model ${ }^{[24]}$ here as the instance feature extractor. The embedding layer ${ }^{[25]}$ was employed to represent bases (15 (A, T, G, C, N, H, B, D, V, R, M, S, W, Y, K) $\rightarrow 4$ (representative dimension)) $\square$ The encoder used a bidirectional RNN structure, which given equal attention to the head and the tail of the instance, and the output was a context vector ${ }^{[26]}$ to represent the feature of the instance. And subsequently, through the MIL layer, the features of all instances were scored and aggregated jointly to determine the type of the bag $^{[20,21,27]}$.

Attention mechanism as the MIL pooling

Reference to previous work of pooling layer structure, we selected the attention-based pooling structure, which exhibited better aggregation and representation capacity ${ }^{[21]}$. It was assumed that the feature extracted by encoder were $\mathrm{C}=\left\{\mathbf{c}_{1}, \ldots, \mathbf{c}_{k}\right\}$, and its corresponding attention weights were $\boldsymbol{\alpha}=\left\{\alpha_{1}, \ldots, \alpha_{k}\right\}$, which could be formulated as follow.

$$
\boldsymbol{\alpha}=\operatorname{softmax}\left(W^{T} \tanh \left(V C^{T}\right)\right)
$$

Where $\mathrm{W} \in \mathrm{R}^{L \times 1}$ and $\mathrm{V} \in \mathrm{R}^{L \times M}$. The attention-base structure allowed to discover the similarity between different instances and made the network have better representability. After the encoder feature was weighted by the attention scores, the probability of determination was output via a sigmoid neuron through a fully connected layer.

Handling of handwritten numbers dataset

The handwritten numbers dataset was used to verify the representational power of the attention score. Each number figure $($ size $=28 \times 28)$ was served as an instance. A bag contained more than 16 instances. For each instance, we treated the image as a sequence containing 28 characters, and each with a representation dimension of 28 , for feeding into the network (Circ-ATTEN-MIL; the embedding layer in encoder block was removed in this task) (Fig.3). A bag is positive when it contains the determining number (Two modes were set: determining number is 0 ; determining number are $0,1,3$ ).

Fusion model: 
The 'weighted feature' (the penultimate layer) of Circ-ATTEN-MIL was extracted as the sequence feature defined by the model. The other features were calculated using the extraction methods of RCM features and conservation features in CircDeep. Combining these three types of features (sequence feature: 100; RCM feature: 40; conservation feature: 23), a four-layer MLP (Multi-Layer Perceptron) network (163-80-20-1 (the output layer is a sigmoid-activated neuron)) was constructed as a fusion model.

\section{Evaluation criteria:}

We evaluated the model performance by classification accuracy, sensitivity, specificity, MCC (Matthews correlation coefficient), and F1 score (formulated as follows).

$$
\begin{aligned}
& \mathrm{MCC}=\frac{T P \times T N-F P \times F N}{\sqrt{(T P+F P)(T P+F N)(T N+F P)(T N+F N)}} \\
& \mathrm{F} 1=\frac{2 \times \text { precision } \times \text { recall }}{\text { precision }+ \text { recall }}
\end{aligned}
$$

\section{Extraction of highly attention sequence splices}

As the attention score was applied to the encoder features of each instance, we assigned the same scores to the sequence of the instance, and collapsed the weighted sequences according to the inverse of the slider rule (Fig.4), and extracted the sequence fragment (with certain length: $>7$ ) with the higher attention score (after scaling to between 0 and 1 : $>0.6$ ), which served as the high attention sequence splices.

Motif enrichment:

MEME software ${ }^{[28]}$ was utilized to perform motif enrichment tasks. In MEME environment, classic mode was selected to enrich motifs in RNA sequences between 6 and 50 lengths (The code is: meme RNA.fasta -rna -nostatus -mod zoops -minw 6 -maxw 50 -objfun classic -markov_order 0).

Result 1: Dataset description

The sequence length distribution and base proportion between circRNAs and other IncRNAs (In training set) were very similar (Fig.5), which illustrated that the simple features between the two-type sequences were comparable and the model feeding with raw sequences was hard to accomplish the identification task by these simple features.

Result2: Model architecture

In instance extraction, the window size was set to 70 , sliding step was set to 5 (Fig.1). In the encoder block, it consists of one embeding_15_4 layer and two bi-direction LSTM_4_150 layers. The final step 
outputs of both directions were concatenated, and via an FCN_300_100 layer, the instance feature (C_100) was obtained. In the attention block, the C_100 features of each instance were accepted as key values. After an FCN_100_30, an FCN_30_1 layer, the dimension for each instance was reduced to 1 (attention value). A softmax layer was utilized to normalize the attention value for each instance, and then the normalized attention score was yield. Finally, the classifier block accepting all instances' weighted C_100 feature, through a fully connected layer and a sigmoid neuron, outputted the identification probabilities (Fig.2).

Result3: Model training and identification evaluation

We used the binary cross-entropy loss function to calculated loss and trained the models with the adam optimization algorithm (learning rate is 0.0002 ; betas $=(0.9,0.999)$; weight decay is $10 \mathrm{e}-5)$. Balancing the accuracy and over-fitness, we chose the model trained at the 70th epoch as the final model and plotted the ROC curve (Fig.6). As a result, the performance of the model training had strong identification efficiency (Train AUC=0.99; Validation AUC=0.97; Test AUC=0.97). Subsequently, multiple evaluation criteria were employed to test the model (Table 1), and these metrics also validated that the model has a high degree of robustness.

Table 1. The evaluation for classification task

\begin{tabular}{|lllllll|}
\hline & Accuracy & Sensitivity & Specificity & Precision & MCC & F1 \\
\hline Train & 0.9552 & 0.9662 & 0.9547 & 0.9713 & 0.9194 & 0.9687 \\
Validation & 0.9333 & 0.9485 & 0.9092 & 0.9433 & 0.8291 & 0.9459 \\
Test & 0.9284 & 0.9396 & 0.9039 & 0.9393 & 0.8435 & 0.9394 \\
\hline
\end{tabular}

Result4: Comparison with other algorithms

This model was compared with ACNN-BLSTM in CircDeep ${ }^{[13]}$, which took the sequence as input without the feature from the secondary structure and conservation score of the sequence. In Circ-ATTEN-MIL, the input was full-length raw sequences. While in ACNN-BLSTM, the input was the padded triplet sequences (the base triplet was transformed to a 40-dimension vector by word2vec; the input length was padded to 8000). The comparison results showed that our final model was better under the three metrics (Table.2). Finally, we incorporated the RCM and conservation features which used in CircDeep model to build a fusion model (Methods), and successfully improved the discriminative power of the final model.

Table 2. The comparison results 


\begin{tabular}{|llll|}
\hline & ACC & MCC & F1 Score \\
\hline PredcircRNA & 0.8056 & 0.6113 & 0.8108 \\
\hline ACNN-BLSTM & 0.8942 & 0.7756 & 0.9149 \\
\hline Circ-ATTEN-MIL & 0.9284 & 0.8435 & 0.9394 \\
\hline CircDeep (fusion) & 0.9327 & 0.8536 & 0.9304 \\
\hline Fusion model & $\mathbf{0 . 9 4 3 4}$ & $\mathbf{0 . 8 7 9 6}$ & $\mathbf{0 . 9 5 4 6}$ \\
\hline
\end{tabular}

Result5: Attention score employed for identifying determining factor

To verifying the representational power of the attention score, we used the handwritten numbers dataset to visualize the known determining factor with the produced attention score. Two model (In encoder block: 2 LSTM_28_10, FCN_10_10; in MIL block: FCN_10_5, FCN_5_1) was trained in this part, one (model 1) with 0 and another (model 2) with 0, 1, 3 as determining number (a bag contains determining number instances was treated as positive sample). The training was stopped after the accuracy exceed 0.90 (around 10 epochs). We visualized the attention score with the matched instances and discovered that the attention score identifies well whether the bag contains a single determinant, multiple identical determinants, or multiple different determinants (Fig.7). Statistics on determining numbers identification showed a very low percentage of false identifications, and although there was a certain unrecognized rate, the identified numbers had a very high confidence level $(>99 \%)$.

Result6: Motif enrichment from high attention sequence

The high attention sequences were extracted for all correct identification circRNAs transcripts. Most of the high attention sequences were between 8-40 in length, and the count of the attention sequences for each transcript was around 4 (Fig.8), which validates our initial assumption that the meaningful features were sparse. All high attention sequences were used for motif enrichment, and multiple validated motifs were yield (Table 3).

Table 3. motif enriched from the sequence

\begin{tabular}{|c|c|c|c|}
\hline Motif & Sequence & E-value & Predicted (Red labelled in sequence: Target loci) \\
\hline 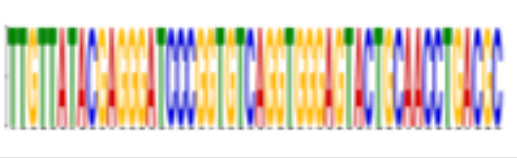 & $\begin{array}{l}\text { TTGTTATACGAGGGATC } \\
\text { CCGGTGTCAGGTGGGA } \\
\text { GTACTGCAACCTGACGC }\end{array}$ & $2.3 e-009$ & $\begin{array}{l}\text { KR super family (autonomous structural domains): Kringle domains are believed to } \\
\text { play a role in binding mediators. (Source: NCBI) }\end{array}$ \\
\hline 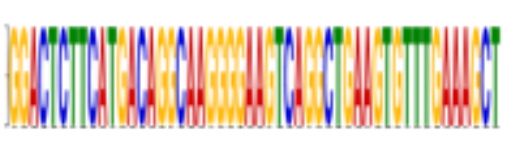 & $\begin{array}{l}\text { GGACTCTTCATGACAGGC } \\
\text { AAGGGGAAGTCAGGCTG } \\
\text { AAGTGITTGAAAGCT }\end{array}$ & $2.1 e+003$ & $\begin{array}{l}\text { SPIl target secuence: May bind RNA and moc̀late urmatured-RNA splicing by } \\
\text { similarity. (Source: JASPAR) }\end{array}$ \\
\hline
\end{tabular}




\begin{tabular}{|c|c|c|c|}
\hline $\mathrm{CCHT}_{2}^{1}$ & $\begin{array}{l}\text { YSSMCCTCCWGGYCCTCC } \\
\text { TGG }\end{array}$ & $6.7 e+004$ & $\begin{array}{l}\text { PLN02915 super family. catalytic subumit. (Source: NCBI) } \\
\text { Contains ETS1 target sequence. (Source JASPAR) }\end{array}$ \\
\hline Wha & $\begin{array}{l}\text { TCCAAGAAACAAAATTTC } \\
\text { CTITAAATTTGACCACTTA } \\
\text { CGGAAAGAAGC }\end{array}$ & $2.0 \mathrm{e}+005$ & $\begin{array}{l}\text { The actual alignment was detected with superfanily member pfann01267. (Source: } \\
\text { NCBI) }\end{array}$ \\
\hline 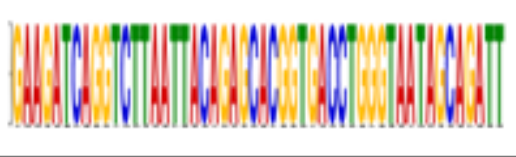 & $\begin{array}{l}\text { GAAGAICAGGTCTTAATTA } \\
\text { CAGAGCACGGTGACCTGG } \\
\text { GTAATAGCAGATT }\end{array}$ & $1.1 \mathrm{e}+006$ & $\begin{array}{l}\text { Contains multi estrogen receptor (ESRl; ESR2) awd estrogen related receptor } \\
\text { (ESRRA) target sequences. (Source: JASPAR and GeneCurdsSuite) }\end{array}$ \\
\hline & CGGCCCCGGGG & $2.1 \mathrm{e}+008$ & $\begin{array}{l}\text { TFAP2E target sequence: may bied to the consensus sequence 5'-GCCNNNGGC-3'. } \\
\text { (Source: JASPAR) }\end{array}$ \\
\hline Ais & $\begin{array}{l}\text { AGTGACAGCAGTTATGAT } \\
\text { AGTAGCAGCA }\end{array}$ & $2.8 \mathrm{e}+007$ & $\begin{array}{l}\text { Contains multi Homebos related factor (A6, A4, B6, C10, C8, D8, B9,B8, B6, B3, } \\
\text { A5, A7, A9, B4, C4, A6) target sequences. (Source: JASPAR and GeneCardsSunte) }\end{array}$ \\
\hline
\end{tabular}

\section{Discussion}

In this project, we designed a deep learning network architecture suitable for learning gene sequence features and implemented the model to accomplish the circRNA identification task. And based on the attention score produced by the model, a large number of key sequence loci for circRNA recognition were extracted. Following the motif enrichment analysis, some possible key motifs for circRNA formation were identified.

The post-transcriptional modifications and a variety of related functions of transcripts are encoded in their sequence ${ }^{[29]}$. Thus, a sequence contains a large number of key loci responsible for each of the processes $^{[30]}$. For machine learning models, which often responsible for discriminating a single function, such as loop formation, the entire sequence can be too redundant and the meaningful features are too sparse. From another viewpoint, the learning-by-sequence task is similar to multiple instance learning $(\mathrm{MIL})^{[20,27,31]}$, that is, for weak label learning problems with sparse features. We changed the convolutional blocks commonly used in the MIL model for feature extraction to an RNN block that is more suitable for sequence learning ${ }^{[32]}$, and used the attention mechanism ${ }^{[21,33]}$, which has stronger representation capability, as the MIL layer. The results demonstrate the validity of the structure and the great potential value of the attention mechanism.

For this circRNA identification task, data were collected from the validated reference sequence with high confidence $^{[22,23]}$. While there are certain problems that the sampling rate was too low. If a single gene is assumed to be a single distribution (which may actually be a set of genes), and the use of a reference sequence causes only one sample to be collected for a single distribution, the sampling rate can be considered to be relatively low. Therefore, if multiple actual sequences can be collected for a single gene, which implies that there may be a variety of mutations in non-relevant features of multiple sequences, while relevant features are more conservative, the increased sampling rate must enhance the model's 
learning of the features and improve its discriminative power. Considering that data collection is more difficult ${ }^{[34]}$, it is worthwhile to explore to improve the effectiveness of the model by trying some data augmentation methods.

The instance is extracted by a moving slider, which can only extract the continuous regional features in the sequence. However, sequences form higher-level stereo structures in space ${ }^{[35]}$, so the key feature can be the combinations of sequences that are far apart. Considering this possibility, adding more mechanisms for instances extraction and combination, to make a single instance can contain multiple combinations of distant sequences, may further improve the discriminative effectiveness as well as the potential representational value of this network structure.

The model can be used for more than just the identification of circRNAs. Since only the original sequence is required as input, the network structure can be used for learning other sequence-related tasks by simply changing the resultant events. Because of its representation capability, it can be used to discover key sequences for different tasks and provide a basis for other relevant research.

\section{Conclusions}

Circ-ATTEN-MIL was designed and used for circRNA identification, and it outperformed other deep learning models currently used. The model utilized the MIL-attention network architecture, which took the complete RNA sequence as input and not only carried out the discriminant probability of circRNA identification, but also outputted the score of the importance of each instance, which could be used for identifying the critical part of a sequence for model judgment and would be able to provide some insights for basic research in related fields.

\section{Declarations}

Ethics approval and consent to participate: Not applicable (No human participation).

Consent for publication: All authors agree to publication.

Availability of data and material: The data and code are available in https://github.com/liuyunho/CircATTEN-MIL, and any other requirement can contact the corresponding author.

Competing interests: No competing interests

Funding: This work was funded by the National Natural Science Foundation of China (Grant Number: 91846302)

Authors' contributions: Conceptualization: Y.L., G.L. and Q.F.; methodology: Y.L., Q.F; network design: Y.L.; validation: Y.L., X.P. and C.Z.; writing-original draft preparation: Y.L. and X.P.; visualization: Y.L. and X.P.; funding acquisition: L.L. 


\section{References}

1. Zhao Z, Sun W, Guo Z, et al. Mechanisms of IncRNA/microRNA interactions in angiogenesis[J]. Life Sci, 2020,254:116900.

2. Zhang X Z, Liu H, Chen S R. Mechanisms of Long Non-Coding RNAs in Cancers and Their Dynamic Regulations[J]. Cancers (Basel), 2020,12(5).

3. Beermann J, Piccoli M T, Viereck J, et al. Non-coding RNAs in Development and Disease: Background, Mechanisms, and Therapeutic Approaches[J]. Physiol Rev, 2016,96(4):1297-1325.

4. Memczak S, Jens M, Elefsinioti A, et al. Circular RNAs are a large class of animal RNAs with regulatory potency[J]. Nature, 2013,495(7441):333-338.

5. Hansen T B, Jensen T I, Clausen B H, et al. Natural RNA circles function as efficient microRNA sponges[J]. Nature, 2013,495(7441):384-388.

6. Hu Z Q, Zhou S L, Li J, et al. Circular RNA Sequencing Identifies CircASAP1 as a Key Regulator in Hepatocellular Carcinoma Metastasis[J]. Hepatology, 2020,72(3):906-922.

7. Miao Q, Zhong Z, Jiang Z, et al. RNA-seq of circular RNAs identified circPTPN22 as a potential new activity indicator in systemic lupus erythematosus[J]. Lupus, 2019,28(4):520-528.

8. Chen L, Zhang Y H, Huang G, et al. Discriminating cirRNAs from other IncRNAs using a hierarchical extreme learning machine (H-ELM) algorithm with feature selection[J]. Mol Genet Genomics, 2018,293(1):137-149.

9. Pan X, Xiong K. PredcircRNA: computational classification of circular RNA from other long noncoding RNA using hybrid features[J]. Mol Biosyst, 2015,11(8):2219-2226.

10. Li J, Zhang X, Liu C. The computational approaches of IncRNA identification based on coding potential: Status quo and challenges[J]. Comput Struct Biotechnol J, 2020,18:3666-3677.

11. Niu M, Zhang J, Li Y, et al. CirRNAPL: A web server for the identification of circRNA based on extreme learning machine[J]. Comput Struct Biotechnol J, 2020,18:834-842.

12. Zhang G, Deng Y, Liu Q, et al. Identifying Circular RNA and Predicting Its Regulatory Interactions by Machine Learning[J]. Front Genet, 2020,11:655.

13. Chaabane M, Williams R M, Stephens A T, et al. circDeep: deep learning approach for circular RNA classification from other long non-coding RNA[J]. Bioinformatics, 2020,36(1):73-80.

14. Ivanov A, Memczak S, Wyler E, et al. Analysis of intron sequences reveals hallmarks of circular RNA biogenesis in animals[J]. Cell Rep, 2015,10(2):170-177.

15. Tomas Mikolov K C G C. Efficient estimation of word representations in vector space[J]. arXiv preprint, 2013:1301-3781.

16. Gajendran S, D M, Sugumaran V. Character level and word level embedding with bidirectional LSTM Dynamic recurrent neural network for biomedical named entity recognition from literature[J]. J Biomed Inform, 2020,112:103609. 
17. Helm M. Post-transcriptional nucleotide modification and alternative folding of RNA[J]. Nucleic Acids Res, 2006,34(2):721-733.

18. Grabherr M G, Haas B J, Yassour M, et al. Full-length transcriptome assembly from RNA-Seq data without a reference genome[J]. Nat Biotechnol, 2011,29(7):644-652.

19. Cui D, Liu Y, Liu G, et al. A Multiple-Instance Learning-Based Convolutional Neural Network Model to Detect the IDH1 Mutation in the Histopathology Images of Glioma Tissues[J]. J Comput Biol, 2020,27(8):1264-1272.

20. Kraus O Z, Ba J L, Frey B J. Classifying and segmenting microscopy images with deep multiple instance learning[J]. Bioinformatics, 2016,32(12):i52-i59.

21. Maximilian Ilse J M T M. Attention-based Deep Multiple Instance Learning[J]. arXiv preprint, 2018:1802-4712.

22. Chen $X$, Han P, Zhou T, et al. circRNADb: A comprehensive database for human circular RNAs with protein-coding annotations[J]. Sci Rep, 2016,6:34985.

23. Frankish $A$, Diekhans $M$, Ferreira $A M$, et al. GENCODE reference annotation for the human and mouse genomes[J]. Nucleic Acids Res, 2019,47(D1):D766-D773.

24. Keneshloo Y, Shi T, Ramakrishnan N, et al. Deep Reinforcement Learning for Sequence-to-Sequence Models[J]. IEEE Trans Neural Netw Learn Syst, 2020,31(7):2469-2489.

25. Hamid M N, Friedberg I. Identifying antimicrobial peptides using word embedding with deep recurrent neural networks[J]. Bioinformatics, 2019,35(12):2009-2016.

26. Zhang B, Xiong D, Xie J, et al. Neural Machine Translation With GRU-Gated Attention Model[J]. IEEE Trans Neural Netw Learn Syst, 2020,31(11):4688-4698.

27. Zhong P, Gong Z, Shan J. Multiple Instance Learning for Multiple Diverse Hyperspectral Target Characterizations[J]. IEEE Trans Neural Netw Learn Syst, 2020,31(1):246-258.

28. Bailey T L, Boden M, Buske F A, et al. MEME SUITE: tools for motif discovery and searching[J]. Nucleic Acids Res, 2009,37(Web Server issue):W202-W208.

29. Boxuan Simen Zhao I A R C. Post-transcriptional gene regulation by mRNA modifications.[J]. Nat Rev Mol Cell Biol, 2017,1(18):31-42.

30. Stage D E, Eickbush T H. Sequence variation within the rRNA gene loci of 12 Drosophila species[J]. Genome Res, 2007,17(12):1888-1897.

31. Carbonneau M A, Granger E, Gagnon G. Bag-Level Aggregation for Multiple-Instance Active Learning in Instance Classification Problems[J]. IEEE Trans Neural Netw Learn Syst, 2019,30(5):1441-1451.

32. Lim D, Blanchette M. EvoLSTM: context-dependent models of sequence evolution using a sequenceto-sequence LSTM[J]. Bioinformatics, 2020,36(Suppl_1):i353-i361.

33. Ashish Vaswani N S N P. Attention Is All You Need[J]. arXiv preprint, 2017:1706-3762.

34. Zirkel A, Papantonis A. Detecting Circular RNAs by RNA Fluorescence In Situ Hybridization[J]. Methods Mol Biol, 2018,1724:69-75. 
35. Miao Z, Westhof E. RNA Structure: Advances and Assessment of 3D Structure Prediction[J]. Annu Rev Biophys, 2017,46:483-503.

\section{Figures}

\section{Instances extracting rule:}

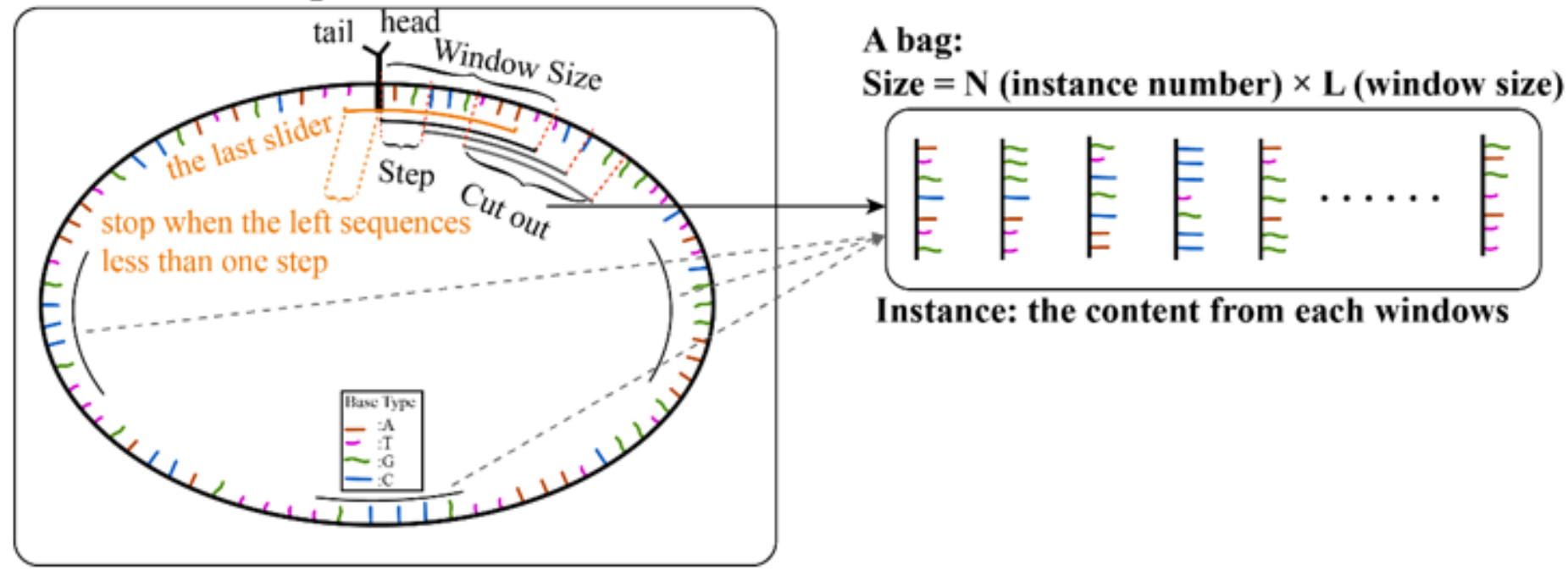

Figure 1

Illustrations of instance extraction from full RNA sequence.

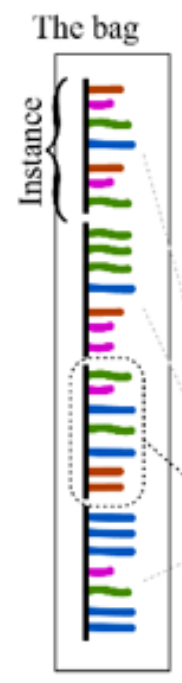

Encoder
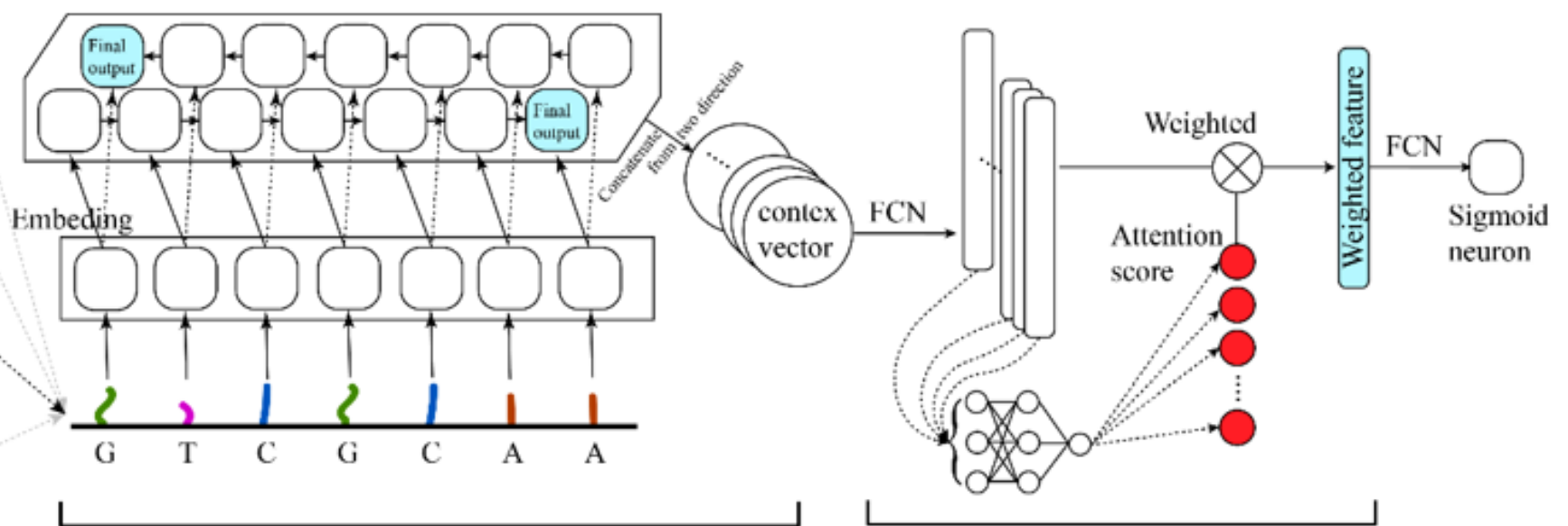

Encoder network

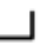

Attention based MIL pooling layer

Figure 2

Illustrations of attention-based deep encoder MIL model structure (Circ-ATTEN-MIL). 


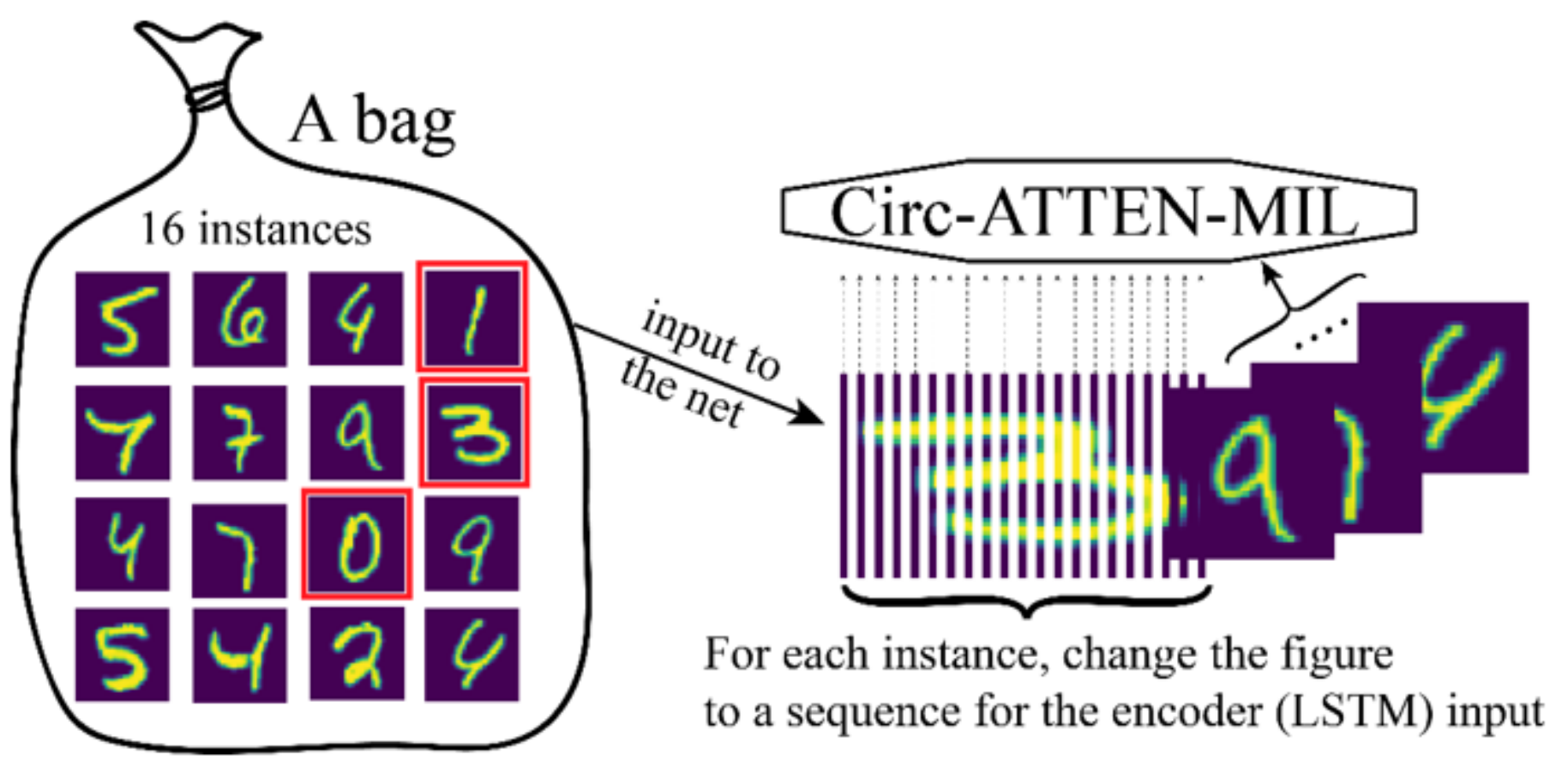

Figure 3

Handling of handwritten numbers dataset for feeding into Circ-ATTEN-MIL.

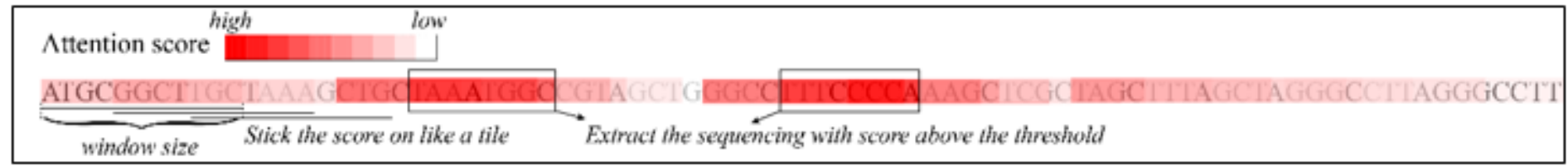

Figure 4

Illustrations of extraction of highly attention sequence splices

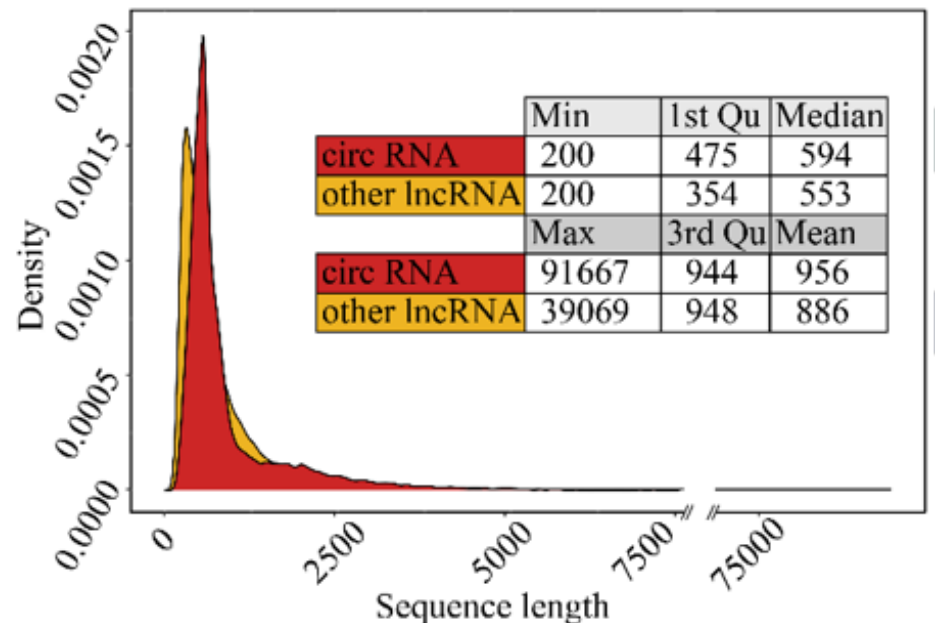

(a)

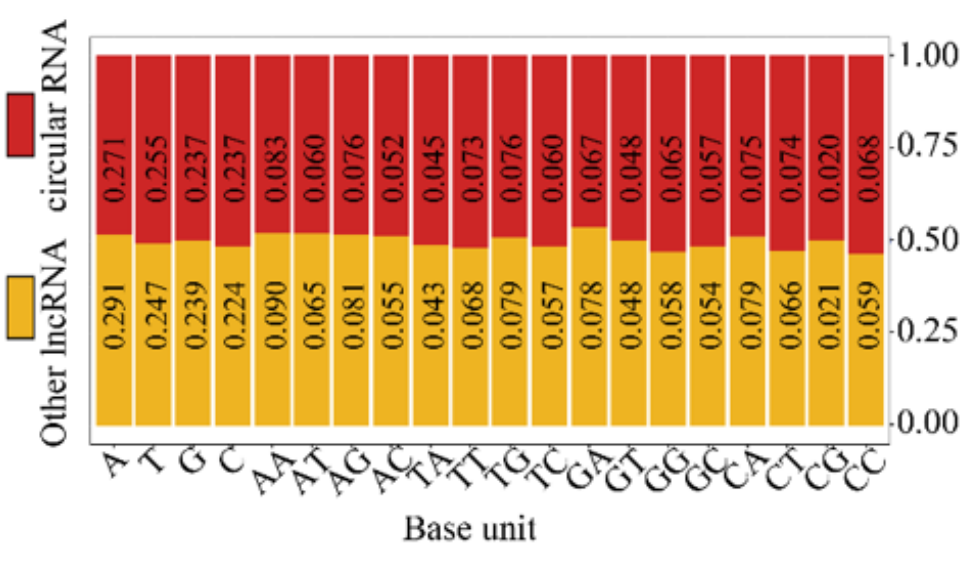

(b) 
Figure 5

The comparison of simple features of sequences between the two-type sequence set: (a). Sequence length distribution comparison. (b). sequence composition comparison.
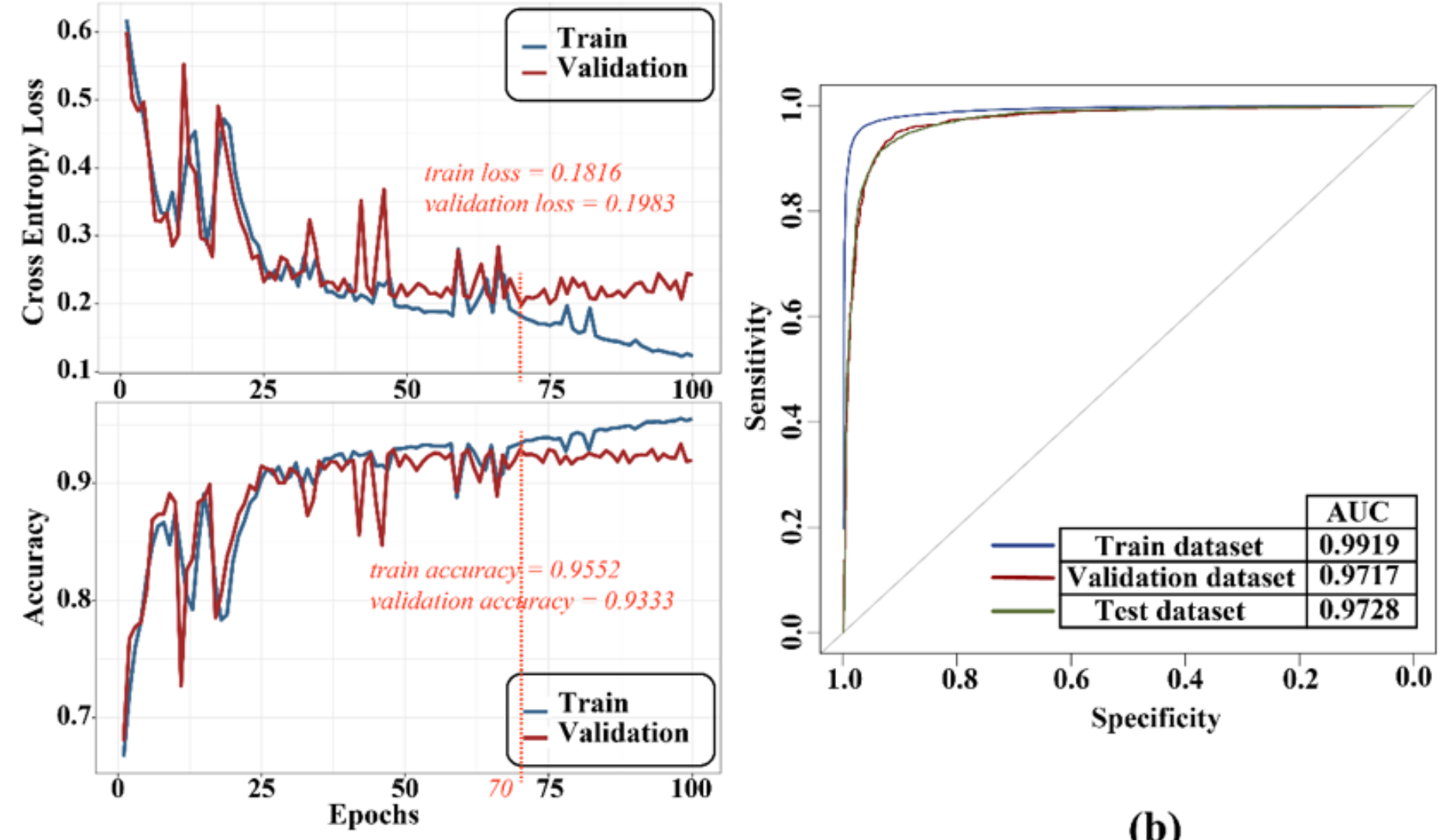

(a)

(b)

\section{Figure 6}

Training process (a) and ROC curve (b). 
(a)

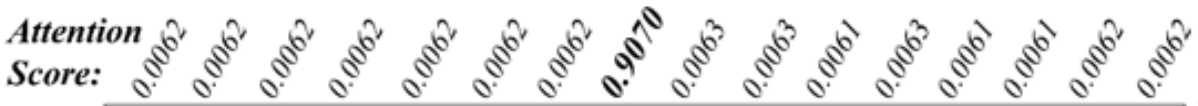
7214149059691597

Bar:

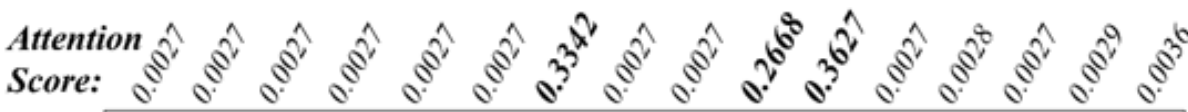

(b) Bag: 4994840240051164 Bar:

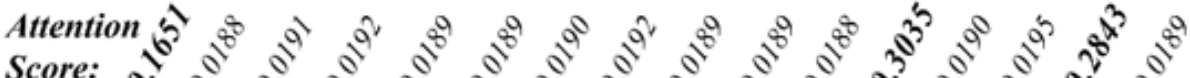

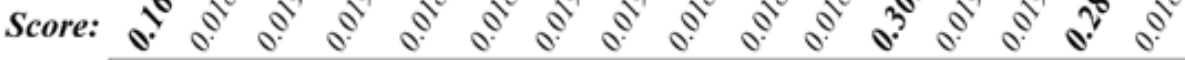

(c)

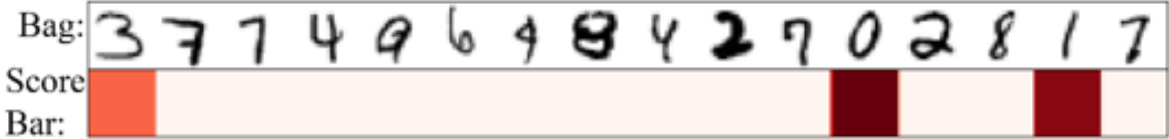

\section{Identification of key factors}

Positive
when bag containing 0

\begin{tabular}{|l|l|}
\hline rightly & wrongly \\
\hline 0.9965 & 0.0035 \\
\hline
\end{tabular}
\begin{tabular}{ll|l|l|l}
0.9965 & 0.0035 \\
\hline
\end{tabular}

Identification of key factors

Positive when bag containing 0

\begin{tabular}{|l|l|l|}
\hline rightly & wrongly & miss- \\
\hline 0.9996 & 0.0004 & 0.0749 \\
\hline
\end{tabular}

Identification of key factors

Positive \begin{tabular}{|l|l|l|}
\hline rightly & wrongly & miss- \\
\hline 0.9928 & 0.0072 & 0.3298 \\
\hline
\end{tabular} when bag containing $0,1,3$

Figure 7

Attention score for identifying the determining numbers. (a). single determinant (model 1); (b). multiple identical determinants (model 1); (c). multiple different determinants (model 2). (left panel: attention score bar; right panel: the rightly and wrongly identify events and miss-identify events).
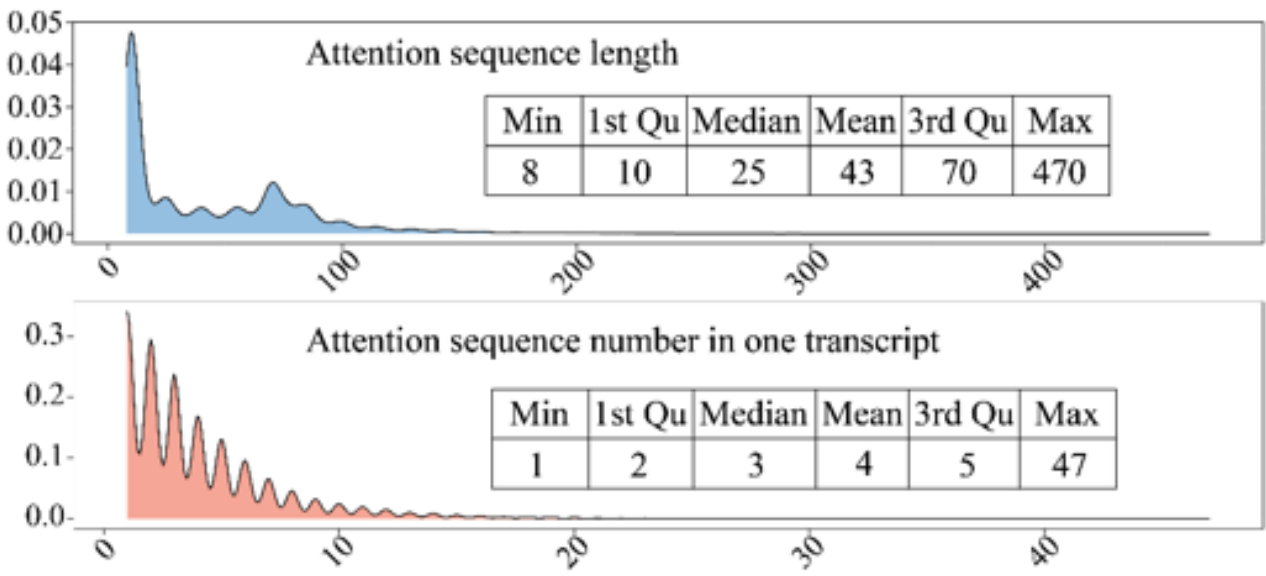

(a)

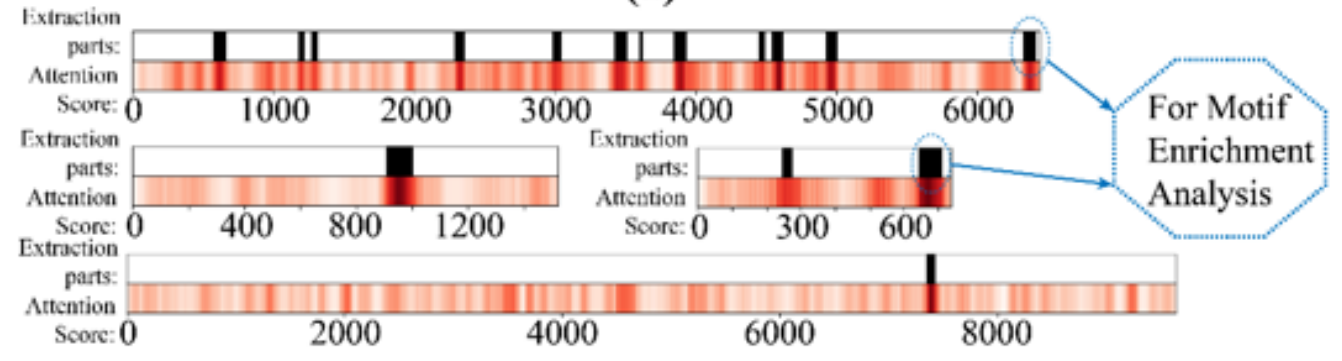

(b)

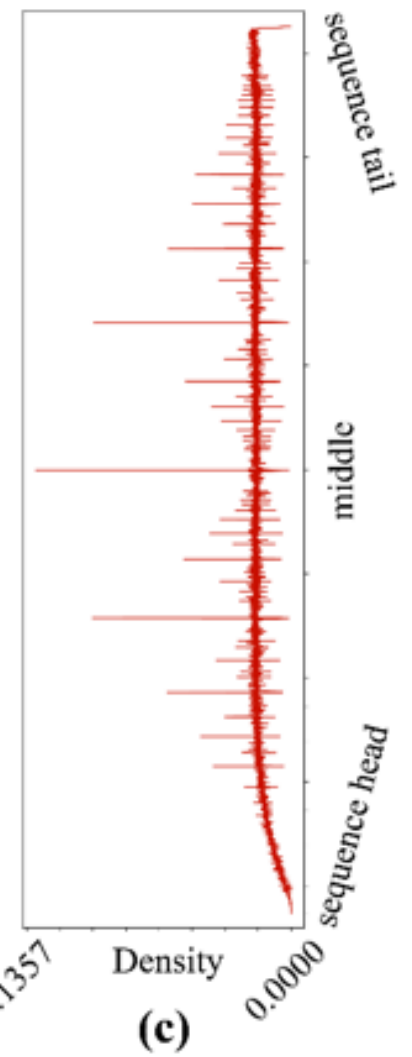

Figure 8 
The high attention sequence distribution. (a) the length distribution (upper) and the attention sequence number for each transcript distribution (lower); (b). the extraction of attention sequence for motif enrichment; (c). density distribution of attention loci on all sequences. 